\title{
Pooling or Fooling? An Experiment on Signaling*
}

\author{
Francesco Feri \\ Royal Holloway University of London \\ Universita di Trieste \\ Giovanni Ponti ${ }^{\dagger}$ \\ Universidad de Alicante \\ The University of Chicago \\ LUISS Guido Carli Roma
}

\author{
Miguel A. Meléndez-Jiménez \\ Universidad de Málaga
}

\author{
Fernando Vega-Redondo \\ Università Bocconi, IGIER
}

\author{
Haihan $\mathrm{Yu}$ \\ Universidad de Alicante
}

\begin{abstract}
This paper reports the evidence from an experiment which takes advantage of the rich informational structure of the so-called Chinos Game, a traditional parlour game played in many countries. In the experiment subjects receive a binary private signal and have to guess the sum of these signals. We compare two constant-sum versions of the Chinos Game. In one version, which we call Preemption Scenario, the first player who guesses right wins the prize. In the alternative version, called the Copycat Scenario, the last player who guesses right wins the prize. While it is straightforward to see that the Preemption Scenario has a unique and fully revealing equilibrium, in all the equilibria of the Copycat Scenario first movers optimally hide their private information. However, our experimental evidence shows that subjects "lie" in the Copycat Scenario (i.e., systematically distort behavior relative to equilibrium play) and they are successful at doing it, despite that benefits from lying are diminishing as the game proceeds.
\end{abstract}

JEL Classification Numbers: C92, D8

KEYWORDS: signaling games, sequential decision-making, insider information disclosure.

${ }^{*}$ We thank Isi Dunietz for his careful reading and precious suggestions. The usual disclaimers apply. Financial support from the Spanish Ministry of Economic Development (ECO2014-52345-P and ECO2015-65820-P), Generalitat Valenciana (Research Projects Grupos 3/086) and Instituto Valenciano de Investigaciones Económicas (IVIE) is gratefully acknowledged.

${ }^{\dagger}$ Corresponding author. Departmento de Fundamentos del Análisis Económico (FAE) - Universidad de Alicante - 03071 Alicante - Spain - e-mail: giuba@ua.es 


\section{Introduction}

The analysis of positional advantages in sequential markets with incomplete (asymmetric) information has been the object of an extensive discussion within the theory of Industrial Organization $\sqrt[1]{1}$ This literature has been enriched by the theoretical and empirical research on strategic information transmission pioneered by Crawford and Sobel (1982), where better informed first movers have the possibility of manipulating the information they hold via the actions they take.2 These issues are of primary importance in a wide variety of economic scenarios -e.g. in financial markets, where traders deal with information as their main economic resource. But other contexts display this same trade-off, such as when competing firms have to decide on the timing of adoption of a new technology -or a specific marketing strategyunder some uncertainty over the underlying technology or consumer preferences.

In all these situations, there are two conflicting considerations faced by early movers:

(i) On the one hand, choosing early on a revealing strategy may have a preemption component, in that it could reduce the strategic possibilities of late movers (Hopenhayn and Squintani, 2011). For instance, in financial markets, traders may want to maximize the chances to buy/sell the right assets in the right moment and, by doing so, eliminate the arbitrage opportunities of later movers.

(ii) On the other hand, early movers may prefer to choose a non-revealing strategy and thus hide their private information. This may happen, for instance, in situations in which firms compete under uncertain demand and early movers may suffer from revealing their private information if the latter is not perfectly correlated with that of their competitors (Gal-Or, 1987).

To analyze these issues, this paper considers a strategic environment that, building upon the related experimental literature, is richer than the received setup but still manageable and intuitive. Specifically, we design an experiment inspired on the 3-player version of the so-called Chinos Game (Feri et al., 2011; Ponti and Carbone, 2009). This is a simple game played by kids in many countries in which players hold a private signal (coins, or pebbles, they hide in their hand) and have to guess, in some pre-specified order, their total sum. At the time a player has to produce her guess, she is informed about her own signal and the guesses of all her predecessors ${ }^{3}$

We study two versions of this game. In one of them, labeled the Preemption Scenario (PS), the winner is set to be the first player whose guess coincides with the sum of signals or, in case no player gets the sum of signals right, the prize goes

1 Gal-Or (1985), for example, shows that first movers have a positional advantage in games with strategic substitutabilities; Rasmusen and Yoon (2012) proves an analogous conclusion when the amount of information first movers hold is only modestly superior than that of their competitors.

${ }^{2}$ There is also another strand of literature that studies positional advantages from a psychological point of view. See, for example, Apesteguia and Palacios-Huerta (2010), Gill and Prowse (2012), Kocher et al. (2012) or Feri et al. (2013).

गThis game was first analyzed theoretically by Pastor-Abia et al. (2001). 
to the last player in the sequence ${ }^{4}$ As the alternative treatment, we consider a second version of the Chinos Game that we call the Copycat Scenario (CS). The CS shares the same game-form of the PS, the only difference being that the winner now coincides with the last player whose guess coincides with the sum of the signals or, in case nobody gets it right, with the first player in line.

However stylized, our experimental setup captures an essential dilemma faced by agents in many signaling situations. To fix ideas, consider the following example.

Example: A fresh "window" for investment opens up in a certain market, associated to some new technology developed elsewhere. (For example, faster Internet access allows new ways to provide entertainment to the household.) A priori, there are a finite number of possible approaches that can be pursued. In practice, however, only one of them is really technologically adequate (or matches sufficiently well consumer preferences). That is, all other firms perform much worse and, for simplicity, we assume that comparably so.

To start with, there are three firms operating in this market. Each of them receives a binary signal in the set $\{0,1\}$, indicating how to address a particular aspect of the problem. In the end, as it turns out, the right investment approach is uniquely characterized by the sum of the three signals received by the firms. In this respect, all three firms are symmetric. There are, however, two other respects in which they are not. On the one hand, they have to make their investment choice in some pre-specified order and this is an important source of asymmetry. On the other, one of them enjoys a dominant position in the market, in the sense that, if no adequate approach is undertaken by any firm, then the dominant firm captures the market with its suboptimal approach.

In principle, one may combine order- and dominance-asymmetry in different ways. Here, for the sake of focus, we consider the following two possibilities, which are those that arguably highlight most starkly the issues involved by balancing preemption and dominance.

PS. The dominant firm moves last and early movers enjoy a preemption advantage, i.e. if a firm develops the right approach first, it captures the whole market.

CS. The dominant firm moves first and late movers enjoy a copycat advantage, i.e. if a firm is the last one to develop the right approach, it captures the whole market.

The two possibilities considered in the above examples provide economic illustrations of the game-theoretical setups that will be formally introduced below. These two games allow us to explore in detail, both theoretically and experimentally, the tension between pooling (i.e., hiding own private information with the aim of acquiring an informational advantage over followers) and fooling (i.e., manipulating

\footnotetext{
${ }^{4}$ In the commonly played Chinos Game, if no player gets the sum of the signals right, the game is repeated afresh. We introduce this modification to the original game-form to avoid across-game strategic considerations that would have substantially complicated the analysis.
} 
own message with the aim of deceive followers) that is the the core of many signaling setups in economics. They help us understand, in particular, how certain off-equilibrium behavior (an attempt to fool the opponents) may naturally arise in the latter case but not in the former, although fooling behavior, per se, cannot be justified on the ground of some equilibrium behavior. Indeed, as we shall see, such an "irrational" behavior is found in our experiment and also pays off, at least in the early instances of repeated play.

A first point to note is that, even though both PS and CS share the same gameform, their contrasting outcome functions dictate completely opposite equilibrium behavior to first movers, Thus, as our theoretical analysis in Section 2.2 will show, while in PS they must fully reveal their private information, in CS they must hide it. In fact, these games are, in a certain sense, symmetric. On the one hand, player 1 in PS and player 3 in CS (we call them the "target players") have exactly the same equilibrium strategy, i.e. to rely on their own signals alone when formulating their guesses.

On the other hand, at equilibrium, the strategies of player 3 in PS and player 1 in CS (we call them the "residual claimants") should carry no informational content about their own signal. This is because player 3's winning chances in PS do not depend on her own action, while player 1 in CS should optimally shade (she can only win if other fail to guess correctly).

Finally, concerning player 2, her intermediate position in the sequence yields the most delicate strategic trade-off between revealing and shading. In PS, "responding to her signal" (and hence revealing it) should be optimal for player 2, but this is subject to the additional consideration that it never pays to repeat player 1's guess. Instead, in CS player 2 faces an even more complicated problem: his optimal guess must involve shading, although a very specific one, i.e. her pooling strategy must maximizes her winning chances compared to any other such strategy.

In summary, PS and CS can be seen as "twin" games in the sense that each player position in one game has a corresponding position in the other. More precisely, they are inverse-twin games because, for players 1 and 3, the symmetric player position in the other game is the opposite one. As we shall see, by comparing the implications of the two game-forms, such inverse-twin feature will help understand the key notion of positional advantage. Note that, in our model, the players who hold a positional advantage in the most clear-cut manner are the target players - i.e. player 1 in PS, and player 3 in CS. This, in turn, is reflected in the fact that, in equilibrium, they play the same strategy and hold the same (highest) winning probability in both games. Indeed, the fact that they enjoy such a high winning probability is also confirmed, at a qualitative level, by our experimental evidence.

The experiments, however, also show significant disparities between actual and equilibrium behavior, as well as in actual and equilibrium winning probabilities. (This will be clearly shown in Figure 1 below.) To anticipate our conclusions in this respect, these can be succinctly summarized as follows.

- The target player in CS (player 3) does better than in PS (player 1), because the former can exploit/decode deviations from equilibrium while the latter cannot. 
- The residual claimants in CS (player 1) and in PS (player 3) do better than predicted at equilibrium because they "passively" benefit from the (unavoidable) mistakes of others.

- The middle player (player 2) does worse in CS than in PS because

(a) she can hardly profit from the mistakes of others in either case, but

(b) her decision is substantially more complex in the CS (and hence worsetailored to available evidence).

In sum, we shall argue that the combined analysis of PS and CS provides an ideal environment to understand signaling in multilateral context. In particular, it can shed light on the tension between pooling and fooling that - because of bounded rationality and the non-equilibrium behavior entailed - plays a key role in so many real-world applications. Conceptually, the notion of fooling is related to that of deception discussed in the literature (Crawford, 2003; Gneezy, 2005; Sobel, 2017), in that it presumes some "model of opponents' mind." Indeed, our experiments show that first movers in the CS often try to fool their followers and they gain by it.

The remainder of the paper is organized as follows. Section 2 provides a brief synopsis of the theory underlying the experiment. In Section 3 we describe the experimental design, while Section 4 summarizes our main results. These include a comparison of the winning probabilities of "twins position" in each game and also the effects of various out-off-equilibrium behaviors, as follows. Our results indicate -among others- that $i$ ) in both games residual claimants win more frequently than in equilibrium -which is already an indirect sign of the relevance of off-equilibrium behavior and that $i i$ ) in CS first-movers successfully fool their followers, but the benefits from strategic manipulation are diminishing as the game proceeds. Finally, Section 5 concludes, followed by Appendices containing the experimental instructions, the derivation of the equilibrium predictions, and further statistical evidence.

\section{The model}

\subsection{Game-form}

Three players, indexed by $i \in N=\{1,2,3\}$, privately receive an iid signal, $s_{i} \epsilon$ $\{0,1\}$, with $s_{i}=1$ with probability $p \in(0,1)$, uniform across players. Players act in sequence, as indicated by their indices, and have to make a guess, $g_{i} \in G=\{0,1,2,3\}$, over the sum of players' private signals, $\sigma=\sum_{i} s_{i}$. By the time player $i$ makes her guess, she is informed of her own signal, $s_{i}$, and the guesses of her predecessors, $g_{j}, j<i$. 


\subsection{Equilibrium prediction}

In what follows, for both PS and CS, we characterize the Perfect Bayesian Equilibrium (PBE) guessing sequences.$^{5}$ By analogy with our experimental conditions, we posit $p>\frac{2}{3}$ (with $p=\frac{3}{4}$ in the experiment). This assumption greatly simplifies the analysis, since the distribution over the sum of $k$ signals (binomially distributed as $\operatorname{Bin}(k, p)$, for $k \leq 2)$ is unimodal. Specifically, if $M_{k}(p)$ is the mode of $\operatorname{Bin}(k, p)$ -i.e., the most likely realization of the sum of $k$ signals- then, for all $p>\frac{2}{3}, M_{1}(p)=1$ and $M_{2}(p)=2$.

In PS the prize goes to the first player who guesses right, i.e., for whom $g_{i}=$ $\sigma$. Otherwise, the prize goes to player 3. Given the realized vector of signals $\left(s_{1}, s_{2}, s_{3}\right)$, let $g^{T}=\left(g_{i}^{T}\right)$ denoting the PBE guessing sequence of treatment $T$, with $T \in\{P S, C S\}$.

Prediction for PS. In PS all PBE share the following guessing sequence, $g^{P S}$ :

$$
g_{1}^{P S}=s_{1}+2, g_{2}^{P S}=g_{1}^{P S}-1 \text { and } g_{3}^{P S} \in G .
$$

For a complete derivation of the PBE, see Appendix B. In words, player 1 has an incentive to maximize her chance to guess right by choosing the fully revealing strategy $g_{1}^{P S}=s_{1}+M_{2}(p)=s_{1}+2$. As for player 2 , if she observes $g_{1}=2$, she then learns that $s_{1}=0$. Thus, in order to maximize her chances to guess right, she should choose $g_{2}=1$ if $s_{2}=0$ and choose $g_{2}=2$ if $s_{2}=1$. However, if she repeats player 1 's choice (i.e., if $g_{2}=2$ ) she gets a null payoff. Therefore, it is also optimal for player 2 to choose $g_{2}=1$ when $s_{2}=1$. Likewise, if player 2 observes $g_{1}=3$, then she learns that $s_{1}=1$. Thus, in order to maximize her chances to guess right, she should choose $g_{2}=2$ if $s_{2}=0$ and $g_{2}=3$ if $s_{2}=1$. However, since she is restricted by the non repetition constraint (i.e., $g_{2}=3$ does not pay), it is also optimal to choose $g_{2}=2$ when $s_{2}=1$. Finally, any possible choice of the residual claimant player 3 is part of an equilibrium, since the payoffs of all players (including herself) do not depend on $g_{3}$.

The equilibrium properties of CS are summarized in the following

Prediction for CS. In CS all PBE share the following guessing sequence, $g^{C S}$ :

$$
g_{1}^{C S} \in G \text { independent of } s_{1}, g_{2}^{C S}=2 \text { and } g_{3}^{C S}=s_{3}+2 \text {. }
$$

For a complete derivation of the PBE, see Appendix B. In words, player 1 wins only if both players 2 and 3 guess wrong. Thus, any pooling strategy by player 1 is consistent with a PBE. As for player 2, she only gets the prize if she guesses right and player 3 does not repeat. Thus, player 2's PBE strategy solves optimally the trade-off between maximizing her winning chances and hiding her own signal to player 3. If $p>2 / 3$, this trade-off is solved by an optimal (pure) pooling strategy, that prescribes $g_{2}^{C S}=2$ independent of $s_{2}$ and $g_{1}$. As for player 3 , since both players

\footnotetext{
B.

${ }^{5}$ A complete description of the PBE equilibria strategies and beliefs can be found in Appendix
} 
1 and 2 pool, in equilibrium she can only condition her play to her own signal and prior probabilities. This, in turn, implies $g_{3}^{C S}=s_{3}+M_{2}(p)$.

Let $w_{i}^{T}$ denote player $i$ 's ex-ante winning probability in treatment $T \in\{P S, C S\}$, conditional of any PBE of the corresponding treatment. As for PS, from (1) it follows that $w_{1}^{P S}=p^{2}, w_{2}^{P S}=2 p(1-p)$ and $w_{3}^{P S}=1-p^{2}-2 p(1-p)$. If $p=3 / 4$ (as in the experiment), then

$$
w_{1}^{P S}=0.56, w_{2}^{P S}=0.38 \text { and } w_{3}^{P S}=0.06 .
$$

From (2) it follows that $w_{1}^{C S}=1-p^{2}-2 p^{2}(1-p) ; w_{2}^{C S}=2 p^{2}(1-p)$ and $w_{3}^{C S}=p^{2}$. If $p=3 / 4$, then

$$
w_{1}^{C S}=0.16, w_{2}^{C S}=0.28 \text { and } w_{3}^{C S}=0.56 \text {. }
$$

Notice that

1. Target players do not rely on others' guesses, but only on their own priors. Therefore, their strategy (and the corresponding winning probability) is exactly the same.

2. Player 2 is better off in PS despite the no repetition constraint, since, in PS, player 1's guess is fully revealing, while in CS it has no informational content. It is player 2 who faces the trade-off between revealing and shading, which is optimally solved in favor of the former (latter) in PS (CS), respectively.

3. This, in turn, implies that, as for residual claimants, player 1 in CS is better off than player 3 in PS.

\section{The experimental design}

\subsection{Sessions}

Four experimental sessions (two sessions per treatment) were run at the Laboratory for Theoretical and Experimental Economics (LaTEx), at the Universidad de Alicante. A total of 96 subjects (24 per session) were recruited among the undergraduate population of the University, mainly, undergraduate students from the Economics Department with no (or very little) prior exposure to game theory. Depending on the session, they played 20 rounds of either CS or PS, between-subjects. The experimental sessions were computerized. Instructions were provided by a selfpaced, interactive computer program that introduced and described the experiment. Subjects were also provided with a written copy of the experimental instructions, identical to what they were reading on the screen ${ }^{6}$

After each round, all subjects were informed of all payoff-relevant information, that is, $i$ ) the signal and choice profiles for all group members and, consequently, ii) the identity of the winner. In addition, they were also provided with a "history

\footnotetext{
${ }^{6}$ The experiment was programmed and conducted with the software $z$-Tree (Fischbacher, 2007). A copy of the instructions, translated into English, can be found in Appendix A.
} 
table" tracking down the sequence of signals and guesses of all group members in previous rounds.

\subsection{Matching}

In all 20 rounds, group composition and role assignment were kept constant. Both these important features of the experimental design were publicly announced at the beginning of each session. In every round, each player's signal was the outcome of an iid random draw with $p=3 / 4$. Given these experimental conditions, we were able to collect, for each treatment, 16 independent observations.

\subsection{Payment}

All monetary payoffs in the experiment are expressed in Spanish Pesetas (SP: 1 $€=166 \mathrm{SP}$ ). All subjects received $1000 \mathrm{SP}$ just for showing up. The fixed prize for each round was set equal to $100 \mathrm{SP}$. Average winnings in the experiment were 1660 SP (i.e., $10 €$ ), for an experiment that lasted, on average, 45 minutes.

\section{Results}

\subsection{Aggregate behavior}

Figure 1 reports the winning frequencies by treatment and player position and compares them with the corresponding equilibrium probabilities. For each player position $i$, let $\hat{w}_{i}^{T}$ be the observed winning frequency in treatment $T \in\{P S, C S\}$, with $\Delta w_{i}^{T}=\hat{w}_{i}^{T}-w_{i}^{T}$ denoting the difference between observed frequencies and equilibrium probabilities.

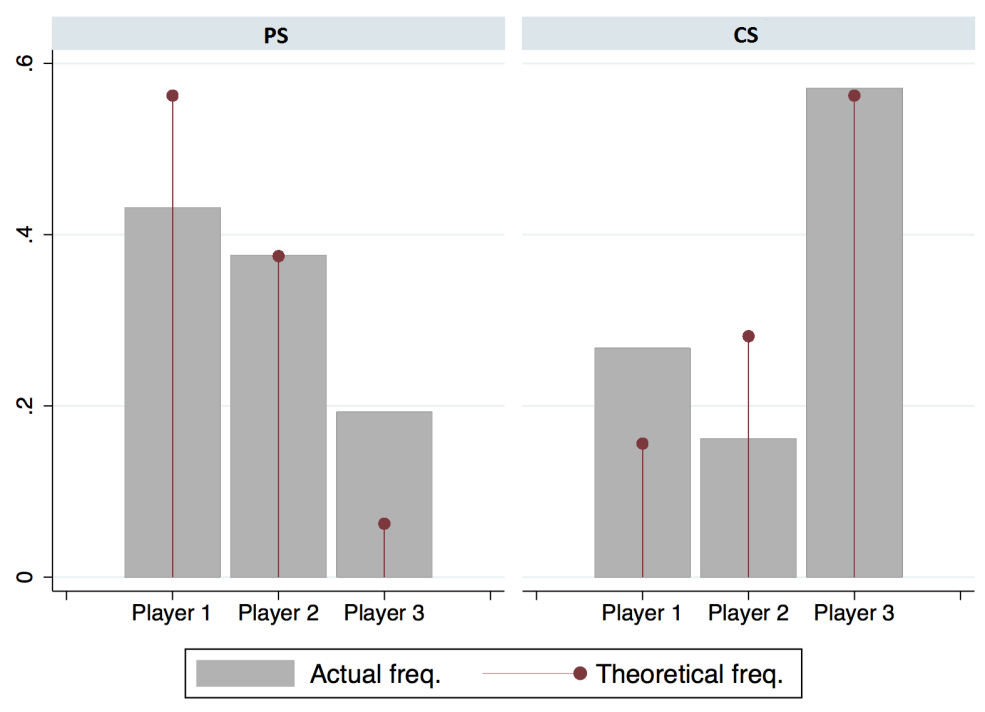

Figure 1: Observed and theoretical winning probabilities 
As for PS, player 1 (3) wins less (more) frequently than in equilibrium (both differences are significant at $1 \%$ confidence using binomial tests), whereas no significant difference is found for player 2. As for CS, it is player 1 who wins at a higher frequency, whereas player 2 wins less (again, these differences are significant at $1 \%$ confidence), while there is no significant difference between observed and predicted winning frequencies for player 3 .

Some considerations are in order at this point. As for target players, along the equilibrium path, both players 1 (3) in PS (CS) should guess based on own private information only: the former because she is the first in line, the latter because both her predecessors should optimally shade their private signal. However, if early movers in CS play off-equilibrium -and, by doing so, partially reveal something about their private signal- players' 3 optimal response consists in exploiting her predecessors' signaling. Whether player 3 in CS can benefit from her predecessors' deviations -and, consequently, gaining a comparative advantage with respect to her "twin player" in PS- depends on her ability to correctly "decode" such deviations.

Along similar lines, also residual claimants may be affected asymmetrically by off-equilibrium play. While in PS player 3 is really "residual", in the sense that she wins the prize independently of her own behavior in that none of her predecessors has guessed right, off-equilibrium signaling from player 1 in CS may, or may not, increase her winning chances. To the extent she is able to "fool" her successors (this is what Sobel (2017) defines as deception), she may outperform her equilibrium winning chances; if, instead, her signaling is correctly decoded by successors, off-equilibrium play may be detrimental.

Finally, comparing the strategic situation of players 2 in PS and CS, remember that the behavior of the former is restricted by the non repetition constraint. By contrast, player 2 in CS is restricted by her successor: if both make the same guess, it is player 3 who gets the prize. Thus, player 2 in CS faces the trade-off between maximizing her winning chances and hiding her own signal from player 3. Moreover, player 2 in CS must also minimize the chances that player 3 repeats her own choice. Thus, the nature of the restriction is inherently more compelling in CS which, in turn, implies that player 2's theoretical winning probability in CS is lower $\left(w_{2}^{P S}=\right.$ 0.37 and $w_{2}^{C S}=0.28$ ).

In what follows, we shall look at Figure 1 with the aim of identifying to which extent off-equilibrium play affects players' performance.

Result 1 (target players). The observed winning frequency of player 3 in CS is significantly higher than that of player 1 in PS (at 5\% confidence, Mann Whitney test), although -in equilibrium- they should be exactly the same.

While player 1 in PS and player 3 in CS are in strategically similar positions, when mistakes occur they are in drastically different ones. No significant differences should be expected in a once-and-for-all play of the game: payoffs should be approximately equal. However, if the game is repeated over time and players can learn the "deviation patterns" of others, player 3 in CS can profit from learning how to decode such patterns, while player 1 in PS cannot. This manifests itself in the fact that player 3 in CS obtains significantly higher payoffs than player 1 in PS. 
In other words, Result 1 indicates that player 3 in CS exploits her positional advantage. This suggests that, in environments where there is conflict of incentives among agents who act sequentially, late-movers may be able to properly use the information obtained from predecessors' mistakes and benefit from it.

Result 2 (residual claimants). In both treatments, residual claimants win more compared with the theoretical prediction. There is no significant difference between $\Delta w_{1}^{C S}$ and $\Delta w_{3}^{P S}$.

Interestingly, both residual claimants -player 3 (1) in PS (CS), respectively- win significantly more with respect to their equilibrium benchmark. This is already an indirect evidence of suboptimal play on behalf of the other group members. Remember that player 3 in PS -contrary to player 1 in CS- does not have any possibility to influence others. Since the theoretical winning probabilities differ across residual claimants $\left(w_{3}^{P S}=0.06\right.$ and $\left.w_{1}^{C S}=0.16\right)$, we compare the differences between observed and theoretical winning frequencies.

Since players' payoff shares deviate significantly from those induced by equilibrium, significant deviations from equilibrium must occur. Who benefits from these deviations? Residual claimants do. This means that these players residual role in terms of the rule of the game (they win when others do not) gets transformed into in an equal share in the residual payoffs that are left on the table when others make mistakes.

Thus, there are no significant differences in the residual claimants' performance, compared with the equilibrium benchmark. However, there may be heterogeneity across groups, so that some players 1 in CS could be more able to exploit their firstmover advantage, compared to others. To check this conjecture, Result 3 compares the performance between the $75 \%$ of best-performing residual claimants in PS and CS.

Result 3 (best-performing residual claimants). For the best performing residual claimants, $\Delta w_{1}^{C S}$ is significantly higher than $\Delta w_{3}^{P S}$ (at $5 \%$ confidence).

Finally, in order to check whether the extra complexity of the restriction in CS hurts player 2 compared to her counterpart in PS, we compare the differences between observed and theoretical frequencies, $\Delta w_{2}$, in the following result.

Result 4 (intermediate players). $\Delta w_{2}^{C S}$ is significantly lower than $\Delta w_{2}^{P S}$ (at $1 \%$ confidence).

As explained in Results 1-3, when mistakes occur, the payoff differences across players in strategically similar positions in CS and PS are essentially due to either differences in learning potential or differences in fooling/revealing potential. Mistakes, however, also bring about an additional consequence: they complicate the analysis of the strategic situation. The equilibrium can no longer be used to predict/understand the behavior of others and, therefore, players must resort to 
decoding systematic patterns from past evidence. The difficulty of this endeavor depends on how sharply defined are the incentives of the agents whose behavioral patterns are to be decoded. In this sense, $P S$ is much simpler than $C S$ : in PS, any player simply wants to guess right. In contrast, in CS, depending on the player position, there are incentives both to guess right -for player 3 and, partially, for player 2- and to hide information -for player 1 and, partially, for player 2. This is why CS is the game where mistakes introduce higher complexity in the analysis. And, given such complexity, the player most affected by it should be player 2, who is simultaneously facing the need to guess right and hide private information. This explains Result 4, which tells us that player 2 is the player whose payoff share falls significantly below the equilibrium prediction.

\subsection{Individual behavior}

This section looks at two complementary informational dimensions, which play different roles in the two treatments.

1. Signaling: the extent to which players' guesses reveal their own private signals. This may appear in two forms: revealing, when own guesses are positively correlated with own signals; or fooling, when own guesses are negatively correlated with own signals.

2. Decoding: the extent of players' ability to gather the private information held by their predecessors.

In principle, both forms of signaling, either revealing or fooling, are suboptimal in CS, as they give followers a chance to decode. By contrast, (no repetition constrained) revealing is the only rational behavior in PS.

As we have just shown, off-equilibrium behavior has asymmetric effects in the two game-forms under scrutiny:

- in PS, if player 1 fails to signal her private information, this may give her followers improved winning chances, although her mistakes might mislead others, giving rise to error cascades (Feri et al., 2011);

- in CS, if player 1 fails to hide her private information (by either revealing, or fooling), this may give her followers improved winning chances conditional on their ability to decode the signaling content of predecessors.

Given these considerations, we analyze subjects' off-equilibrium behavior by way of two complementary methods:

1. Correlation method. We compute $i$ ) the correlation between subjects' own private signals and guesses and $i i$ ) the correlation between own guesses and those of predecessors. The former is a proxy of the signaling content of guesses; the latter measures the extent of decoding. 
2. Actions classification method. We first partition actions according to their signaling content. In PS we look at the consequences of player 1's deviation from her (fully revealing) optimal strategy; in CS we distinguish between revealing and fooling on behalf of player 1 and look at the consequences of these alternative behaviors on winning frequency profiles.

In what follows, we shall report the results of the correlation methods. The analysis of the action classification method (to be considered as a robustness check) can be found in Appendix B. There we find that $i$ ) in PS off-equilibrium play from player 1 mostly favors player 2 and is reduced as the game proceeds and $i i$ ) in CS fooling behavior from player 1 is successful, mostly at the expense of player 3 although also this phenomenon is drastically reduced in the last repetitions of the game.

Let $c\left(g_{i}, s_{i}\right)\left(c\left(g_{i}, g_{j}\right), i<j\right)$ denote, respectively, the correlation coefficients between own guesses and signals (own and predecessors' guesses) evaluated across the 20 rounds of play, where the former is a proxy of the degree with which a subject reveals her private signal and the latter captures the dependence of followers' guesses on the information revealed by predecessors. Table 1 reports the estimated coefficients of some OLS regressions,

$$
\operatorname{pwin}_{i}=\alpha+\sum_{i} \beta_{1}^{i} c\left(g_{i}, s_{i}\right)+\sum_{i} \sum_{j<i} \beta_{2}^{i, j} c\left(g_{i}, g_{j}\right)+u_{i t},
$$

where the dependent variable, $\mathbf{p w i n}_{i}$, is the relative frequency of winning rounds for the player $i$ of some matching group. The most interesting fact is that, in CS, the winning probability of player 1 (3) is decreasing (increasing) in $c\left(g_{1}, s_{1}\right)$, respectively. This result shows that player 3 gets hurt if player 1 fools.

Table 1: OLS regression of winning frequencies on correlations coefficients

\begin{tabular}{l|lll|lll}
\hline & PS & & & CS & & \\
\hline VARS. & pwin $_{1}$ & pwin $_{2}$ & pwin $_{3}$ & pwin $_{1}$ & pwin $_{2}$ & pwin $_{3}$ \\
\hline$c\left(g_{1}, s_{1}\right)$ & $0.144^{* *}$ & -0.12 & -0.024 & $-0.196^{*}$ & -0.124 & $0.319^{* *}$ \\
& $(0.052)$ & $(0.098)$ & $(0.1)$ & $(0.096)$ & $(0.084)$ & $(0.127)$ \\
$c\left(g_{2}, s_{2}\right)$ & 0.016 & 0.08 & -0.096 & -0.099 & 0.071 & 0.028 \\
& $(0.065)$ & $(-0.122)$ & $(0.124)$ & $(-0.086)$ & $(0.075)$ & $(0.114)$ \\
$c\left(g_{3}, s_{3}\right)$ & -0.002 & 0.205 & -0.204 & $-0.263^{* *}$ & -0.171 & $0.434^{* *}$ \\
& $(0.091)$ & $(0.172)$ & $(0.175)$ & $(0.109)$ & $(0.096)$ & $(0.145)$ \\
$c\left(g_{2}, g_{1}\right)$ & -0.01 & 0.005 & 0.005 & 0.033 & -0.033 & 0 \\
& $(0.048)$ & $(0.092)$ & $(0.093)$ & $(0.074)$ & $(0.065)$ & $(0.098)$ \\
$c\left(g_{3}, g_{1}\right)$ & 0.061 & 0.262 & $-0.323^{*}$ & -0.024 & 0.047 & -0.023 \\
& $(0.082)$ & $(0.156)$ & $(0.158)$ & $(0.093)$ & $(0.081)$ & $(0.123)$ \\
$c\left(g_{3}, g_{2}\right)$ & -0.13 & -0.161 & $0.291^{*}$ & 0.024 & $-0.402^{* * *}$ & $0.378^{*}$ \\
& $(0.075)$ & $(0.142)$ & $(0.144)$ & $(0.128)$ & $(0.112)$ & $(0.17)$ \\
Constant & $0.352^{* * *}$ & $0.374^{* * *}$ & $0.274^{* *}$ & $0.425^{* * *}$ & $0.368^{* * *}$ & 0.207 \\
& $(0.055)$ & $(0.104)$ & $(0.106)$ & $(0.089)$ & $(0.078)$ & $(0.119)$ \\
\hline Obs. & 16 & 16 & 16 & 16 & 16 & 16 \\
\hline
\end{tabular}

Robust standard errors in parentheses. ${ }^{*} p<0.10,{ }^{* *} p<0.05,{ }^{* * *} p<0.01$ 
As Table 1 shows, while in PS the coefficient of $c\left(g_{1}, s_{1}\right)$ is positive (this indicating that revealing pays off for player 1$)$, the reverse occurs in CS. In addition, in CS the impact of $c\left(g_{1}, s_{1}\right)$ on pwin $_{3}$ is positive and highly significant. We are interested in determining whether these results from CS are due to the fact that fooling behavior is successful (harmful) for player 1 (player 3), rather than revealing is harmful (successful) for player 1 (player 3), respectively. With this objective, Table 6 in Appendix B decomposes the effect of $c\left(g_{1}, s_{1}\right)$ into two components, depending on whether it is positive or negative. As Table 6 shows, we see that fooling has an impact on winning prospects of players 1 and 3 while revealing has not.

\section{Conclusion}

This paper builds upon the intriguing structure of a parlor game that enriches the strategic content of classic models of signaling. The comparison between PS and its "opposite twin", CS, unravels, in its simplicity, the tension between signaling and shading, between pooling and fooling. If we focus on target players (i.e., those who enjoy a positional advantage) and residual claimants (i.e., those who suffer a positional disadvantage), in PS there are no confounding factors determining how players' performance is affected by off-equilibrium behavior. This is due to the fact that the target player 1 exclusively suffers from her own deviations (regardless of others' choices) and the residual claimant player 3 exclusively benefits from others' deviations (regardless of her own choice). By contrast, in CS the effects of outof-equilibrium behavior for targets and residual claimants are more intricate: in addition from suffering from her own deviations, the target player 3 may either benefit or be harmed by predecessors' deviations, to the extent to which she is able to properly decode or is fooled. Relatedly, deviations from equilibrium of the residual claimant player 1 can also affect her performance, either negatively (if successors are, to some extent, able to infer her private information) or positively (if she is able to fool her followers). In this sense, PS may be conceived as a benchmark and the comparison of CS with PS allows us to study whether (out-of-equilibrium) manipulation pays off.

In this respect, the relative success of fooling behavior in CS, against the equilibrium prediction, is somewhat disturbing for game theory and can only be interpreted as the consequence of some "common knowledge of irrationality" on behalf of the players, together with the fact that, for the residual claimant player 1, fooling is relatively "cheap" in that her objective is fooling the others rather than being the only one guessing right (Crawford, 2003; Gneezy et al., 2016). This consideration notwithstanding, it is important to notice that, in our experimental setting, fooling pays off in CS, although only in the short-run. This pattern confirms the findings of Forsythe et al. (1999) in that buyers who are most frequently lied to are less gullible in the continuation of the experiment, although subjects who observe that others are particularly gullible, do not exploit the observation with more lying.

Overall, we find that first movers' gains from fooling are offset by their positional disadvantage and that followers are more successful in decoding prior messages as the game proceeds. This may confine fooling as a short-run phenomenon. Additionally, the asymmetric effects of fooling and revealing strategy echoes the results of Sutter 
(2009), suggesting that also truthtelling might be a tool for strategic manipulation.

In sum, our simple games of incomplete information embody -in a stylized setupthe incentives to signal and shade one's own private information that arise in most interesting applications. In this respect, Result 2 plays, essentially, a reassuring role: residual players in each game are symmetrically affected by mistakes. The main insights then follow from Results 1, 3 and 4, all of which highlight a separate important factor: Result 1 centers on coding, Result 3 on fooling, and Result 4 on complexity. To understand their role, we have considered pairs of agents who are placed in strategically similar positions in each game, so that their respective behavior predicted at a (mistake-free) equilibrium is similar as well. Admittedly, the factors highlighted in our analysis are particularly stark because of the equally stark contrast displayed by the two games under consideration. However, the same three factors should be at play in more complicated games as well.

\section{References}

Apesteguia, J. and Palacios-Huerta, I. (2010). Psychological pressure in competitive environments: Evidence from a randomized natural experiment. American Economic Review, 100 (5), 2548-2564.

Crawford, V. P. (2003). Lying for strategic advantage: rational and boundedly rational misrepresentation of intentions. American Economic Review, 93, 133-149.

Crawford, V. P. and Sobel, J. (1982). Strategic information transmission. Econometrica, 50 (6), 1431-1451.

Feri, F., Innocenti, A. and Pin, P. (2013). Is there psychological pressure in competitive environments? Journal of Economic Psychology, 39, 249-256.

Feri, F., Meléndez-Jiménez, M. A., Ponti, G. and Vega-Redondo, F. (2011). Error cascades in observational learning: An experiment on the chinos game. Games and Economic Behavior, 73 (1), 136-146.

FischBACHER, U. (2007). z-tree: Zurich toolbox for ready-made economic experiments. Experimental economics, 10 (2), 171-178.

Forsythe, R., Lundholm, R. and Rietz, T. (1999). Cheap talk, fraud, and adverse selection in financial markets: Some experimental evidence. Review of Financial Studies, 12 (3), 481-518.

GAL-Or, E. (1985). First mover and second mover advantages. International Economic Review, 26(3), 649-653.

GAL-Or, E. (1987). First mover disadvantages with private information. Review of Economic Studies, 54(2), 279-292.

Gill, D. and Prowse, V. (2012). A structural analysis of disappointment aversion in a real effort competition. American Economic Review, 102 (1), 469-503. 
Gneezy, U. (2005). Deception: The role of consequences. American Economic Review, 95 (1), 384-394.

Gneezy, U., Kajackaite, A. And Sobel, J. (2016). Lying aversion and the size of the lie, University of California San Diego, mimeo.

Hopenhayn, H. A., and Squintani, F. (2011). Preemption games with private information. Review of Economic Studies, 78, 667-692.

Kocher, M., Lenz, M. and Sutter, M. (2012). Psychological pressure in competitive environments: New evidence from randomized natural experiments. Management Science, 58 (8), 1585-1591.

Pastor-Abia, L., Pérez-Jordá, J. M., San-Fabián, E., Louis, E. and VegaRedondo, F. (2001). Strategic behavior and information transmission in a stylized (so-called chinos) guessing game. Advances in Complex Systems, 1, 177-190.

Ponti, G. and Carbone, E. (2009). "Positional learning with noise". Research in Economics, 63 (4), 225-241.

Rasmusen E., and Yoon, Y. R. (2012). First versus second mover advantage with information asymmetry about the profitability of new markets. The Journal of Industrial Economics, 60(3), 374-404.

Sobel, J. (2017). Lying and deception in games, University of California San Diego, mimeo.

SutTer, M. (2009). Deception through telling the truth? Experimental evidence from individuals and teams. The Economic Journal, 119 (534), 47-60. 


\section{Appendix A}

\section{A1. Experimental Instructions}

\section{Part of the instructions common to PS and CS:}

Welcome to the experiment! This is an experiment to study how people solve decision problems. Our unique goal is to see how people act on average; not what you in particular are doing. That is, we do not expect any particular behavior of you. However, you should take into account that your behavior will affect the amount of money you will earn throughout the experiment. These instructions explain the way the experiment works and the way you should use your computer. Please do not disturb the other participants during the course experiment. If you need any help, please, raise your hand and wait quietly. You will be attended as soon as possible.

How to get money! This experimental session consists of 20 rounds in which you and two additional persons in this room will be assigned to a group, that is to say, including you there will be a total of three people in the group. You, and each of the other two people, will be asked to make a choice. Your choice (and the choices of the other two people in your group) will determine the amount of money that you will earn after each round. Your group will remain the same during the whole experiment. Therefore, you will be always playing with the same people. During the experiment, your earnings will be accounted in pesetas. At the end of the experiment you will be paid the corresponding amount of Euros that you have accumulated during the experiment.

The game. Notice that you have been assigned a player number. Your player number is displayed at the right of your screen. This number represents your player position in a sequence of 3 (Player 1 moves first, Player 2 moves after Player 1 and Player 3 moves after Players 1 and 2). Your position in the sequence will remain the same during the entire experiment. At the beginning of each round, the computer will assign to each person in your group (including yourself) either 0 tokens or 1 token. Within each group, each player is assigned 0 tokens with a probability of $25 \%$ and is assigned 1 token with a probability of $75 \%$. The fact that a player is assigned 0 tokens or 1 token is independent of what other players are assigned; that is to say, the above probabilities are applied separately for each player.

At each round, the computer executes again the process of assignment of tokens to each player as specified above. The number of tokens that each player is assigned at any particular round does not depend at all on the assignments that he/she had in other rounds. You will only know the number of tokens that you have been assigned (0 or 1), and you will not know the number of tokens assigned to the other persons in your group. The same rule applies for the other group members (each of them will only know his/her number of tokens).

At each round you will be asked to make a guess over the total number of tokens distributed among the three persons in your group (including yourself) at the current round. The other members of your group will also be asked to make the same guess. The order of the guesses corresponds to the sequence of the players in the group. 
That is to say: Player 1 makes his/her guess first, then Player 2 makes his/her guess and, finally, Player 3 makes his/her guess. Moreover, you will make your guess knowing the guesses of the players in your group that moved before yourself. Therefore, Player 2 will know Player 1's guess and Player 3 will know both Player 1 and Player 2's guesses.

At each round there is a unique prize of 50 pesetas that will be assigned to one player of the group. The remaining players will earn nothing.

\section{Part of the instructions specific of PS:}

The rule for assigning the prize in the group is as follows: (i) If for one or more players of the group, the guess coincides with the total number of tokens distributed in the group, the prize is assigned to the first player in the sequence who guessed the total number of tokens. (ii) If there is no player whose guess coincides with the total number of tokens in the group, the prize is assigned to Player 3.

Let us see examples of case (i): If all the three players guess the total number of tokens, the prize is assigned to Player 1. If only Players 2 and 3 guess the total number of tokens, the prize is assigned to Player 2. Obviously, if only one player guesses the total number of tokens, the prize is assigned to her.

\section{Part of the instructions specific of CS:}

The rule for assigning the prize in the group is as follows: (i) If for one or more players of the group, the guess coincides with the total number of tokens distributed in the group, the prize is assigned to the last player in the sequence who guessed the total number of tokens. (ii) If there is no player whose guess coincides with the total number of tokens in the group, the prize is assigned to Player 1.

Let us see examples of case (i): If all the three players guess the total number of tokens, the prize is assigned to Player 3. If only Players 1 and 2 guess the total number of tokens, the prize is assigned to Player 2. Obviously, if only one player guesses the total number of tokens, the prize is assigned to her. 


\section{Appendix B}

\section{B1. Theory: Perfect Bayesian Equilibria (PBE)}

We focus on behavioral strategies, defined in the conventional fashion as a mapping from information sets to (possibly probabilistic) choices. Let $\mathcal{H}_{i}$ denote the collection of player $i$ 's information sets. For player 1, we can simply write $\mathcal{H}_{1} \equiv\left\{h=s_{1}: s_{1}=0,1\right\}$, since she has only two information sets that can be associated to each of the possible realizations of $s_{1}$. For players 2 and 3 , information sets can be defined as $\mathcal{H}_{2} \equiv\left\{h=\left(g_{1}, s_{2}\right)\right\}$ and $\mathcal{H}_{3} \equiv\left\{h=\left(g_{1}, g_{2}, s_{3}\right)\right\}$, respectively. Player $i$ 's behavioral strategy is denoted by $\gamma_{i}: \mathcal{H}_{i} \rightarrow \Delta(G)$, where $\gamma_{i}^{h}\left(g_{i}\right)$ stands for the probability of choosing $g_{i}$ at information set $h$.

Next, we define players' beliefs as systems of probabilities of signals conditional on choices. Given that signals are iid and choices are publicly observed, we make the simplifying assumption that later movers hold common beliefs of previous signals. First, we have the system $\left\{\mu^{1}\left(g_{1}\right)\right\}_{g_{1} \in G}$, where $\mu^{1}\left(g_{1}\right) \in[0,1]$ is the probability associated (by players 2 and 3) to $s_{1}=1$ when the choice of player 1 has been $g_{1}$. Analogously, we have $\left\{\mu^{2}\left(g_{1}, g_{2}\right)\right\}_{g_{1}, g_{2} \in G}$, where $\mu^{2}\left(g_{1}, g_{2}\right) \in[0,1]$ is the probability associated (by player 3) to $s_{2}=1$ when the choices of players 1 and 2 have been $g_{1}$ and $g_{2}$, respectively.

\section{Perfect Bayesian Equilibrium of the Preemption Scenario}

Since in PS player 3's behavior is irrelevant, let us define the PBE focusing on $\gamma_{1}, \gamma_{2}$ and $\left\{\mu^{1}\left(g_{1}\right)\right\}_{g_{1} \in G}$. Let $p>2 / 3$. In a PBE of the PS, the following conditions must hold:

$$
\begin{aligned}
& \gamma_{1}^{\left(s_{1}\right)}\left(s_{1}+2\right)=1 \text { for all } s_{1} \in\{0,1\} \\
& \gamma_{2}^{\left(g_{1}, s_{2}\right)}\left(g_{1}-1\right)=1 \text { for all } g_{1} \geq 2 \text { and } s_{2} \in\{0,1\} \\
& \mu^{1}(2)=0, \mu^{1}(3)=1
\end{aligned}
$$

Out of the PBE equilibrium path, i.e. when $g_{1}<2, \mu^{1}\left(g_{1}\right)$ is unrestricted. Depending on the specific values adopted for such belief, the corresponding $\gamma_{2}^{\left(g_{1}, s_{2}\right)}($. would follow. For completeness, we shall construct a complete PBE of the PS combining (4) and (5):7

$$
\begin{aligned}
& \gamma_{2}^{\left(0, s_{2}\right)}\left(s_{2}+1\right)=1 \text { for all } s_{2} \in\{0,1\} \\
& \gamma_{2}^{(1,0)}(0)=\gamma_{2}^{(1,1)}(2)=1 \\
& \mu^{1}\left(g_{1}\right)=0 \text { for all } g_{1}<2 .
\end{aligned}
$$

\section{Perfect Bayesian Equilibrium of the Copycat Scenario}

Let $p>2 / 3$. In a PBE of the CS, the following conditions must hold:

\footnotetext{
${ }^{7}$ Here we are using the same (out of equilibrium) belief criterion as Feri et al. $(2011)$ : if a player plays suboptimally, successors believe that she has the signal that, conditional on her choice, minimizes losses.
} 


$$
\begin{aligned}
& \gamma_{1}^{(0)}\left(g_{1}\right)=\gamma_{1}^{(1)}\left(g_{1}\right) \text { for all } g_{1} \in G \\
& \gamma_{2}^{\left(g_{1}, s_{2}\right)}(2)=1 \text { for all } g_{1} \in G \text { and } s_{2} \in\{0,1\} \\
& \gamma_{3}^{\left(g_{1}, g_{2}, s_{3}\right)}\left(s_{3}+2\right)=1 \text { for all } g_{2} \geq 2 \text { and } s_{3} \in\{0,1\} \\
& \mu^{1}\left(g_{1}\right)=p \text { for all } g_{1} \in G \\
& \mu^{2}\left(g_{1}, 2\right)=p \text { for all } g_{1} \in G \\
& \mu^{2}\left(g_{1}, 3\right) \geq p /(3 p-1) \text { for all } g_{1} \in G
\end{aligned}
$$

Note that, in order to have an equilibrium, it is necessary that player 3 believes, with a sufficiently high probability, that player 2 is rational: That she does not choose $g_{2}=3$ when $s_{2}=0$. When $g_{2}<2, \mu^{2}\left(g_{1}, g_{2}\right)$ is unrestricted. Depending on the specific values adopted for such belief, the corresponding $\gamma_{3}^{\left(g_{1}, g_{2}, s_{3}\right)}($.$) would follow.$ For completeness, we shall construct a complete PBE of the CS combining (6) and (7) 8

$$
\begin{aligned}
& \gamma_{3}^{\left(g_{1}, g_{2}, s_{3}\right)}\left(s_{3}+1\right)=1 \text { for all } g_{2}<2 \text { and } s_{3} \in\{0,1\} \\
& \mu^{2}\left(g_{1}, g_{2}\right)=0 \text { for all } g_{2}<2 .
\end{aligned}
$$

\section{B2. Additional statistical evidence: action classification method}

We now look at the effects of off-equilibrium behavior of player 1 on players' winning chances and the resulting learning dynamics using the action classification method. In PS we look at the consequences of player 1's deviation from her optimal fully revealing strategy; in CS we focus on the effects, on behalf of player 1, of using a signaling strategy, either revealing or fooling.9 In both cases, we look at the full dataset first and then we split it into the first (last) ten rounds, in search of possible learning effects.

\section{- PS: Is my predecessor's mistake a curse or a bless?}

As we know from the prediction for PS, player 1 has a unique -and relatively simple- optimal guess, which consists of adding 2 to her private signal. We find that $54 \%$ (172/320) of choices of player 1 in PS fits this criterion. At the individual level, the relative frequency of adoption of the equilibrium strategy for the 16 subjects acting as player 1 in PS range from $35 \%$ to $70 \%$, with a median of $53 \%$. Figure 2 tracks the relative frequency with which player 1 deviates from the equilibrium strategy across rounds. As Figure 2 shows, average trend is decreasing, but suboptimal play does not seem to vanish as the experiment reaches the end.

We estimate the following random-effect linear probability model:

$$
P\left(y_{i t}=1\right)=\alpha+\beta x_{1 t}+u_{i t},
$$

\footnotetext{
${ }^{8}$ It can be shown that, independently of the value of $\mu^{2}\left(g_{1}, g_{2}\right)$ for each $g_{2}<2$ and the corresponding best response of player $3\left(\gamma_{3}^{\left(g_{1}, g_{2}, s 3\right)}\right)$, player 2 never finds it profitable to deviate to choose $g_{2}<2$.

${ }^{9}$ Obviously, when looking at an individual action -as opposed to the full sequence- we cannot define a pooling strategy. As a consequence, it may well happen that some actions we classify as part of a revealing plan (or fooling) plan are indeed part of a pooling strategy. We are well aware of this limitation of the action classification method, although the latter can really take advantage of the panel structure of our dataset, something which is completely neglected by our correlation method.
} 


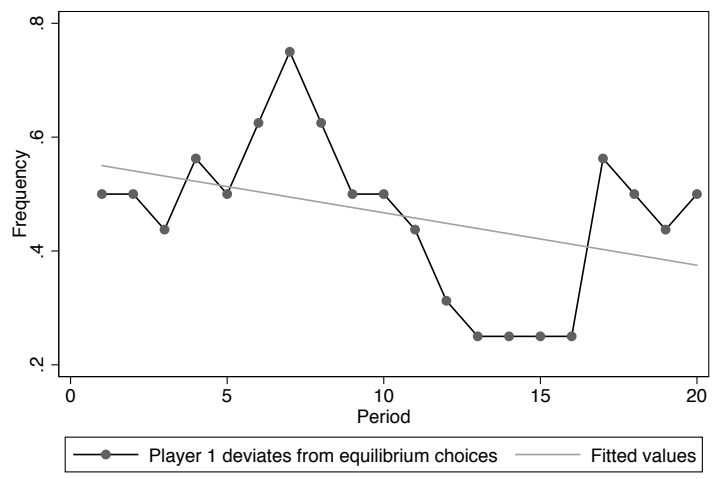

Figure 2: Player 1's off-equilibrium behavior in PS

where $y_{i t}=1$ if player $i$ wins the prize in round $t$ and $x_{1 t}=1$ if player 1 deviates from the equilibrium strategy (11). Table 2 reports the estimated coefficients using the full sample. As Table 2 shows, player 2 significantly benefits from player 1's deviation (at the 1\% level), while the same result does not hold for player 3.

Table 2: Regression of winning probability on specific strategy in PS

\begin{tabular}{lccc}
\hline \hline & Player1 & Player2 & Player3 \\
\hline Player 1 deviates & $-0.325^{* * *}$ & $0.285^{* * *}$ & 0.058 \\
& $(0.050)$ & $(0.054)$ & $(0.050)$ \\
& & & \\
Constant & $0.581^{* * *}$ & $0.256^{* * *}$ & $0.154^{* * *}$ \\
& $(0.031)$ & $(0.028)$ & $(0.033)$ \\
\hline Number of Obs & 320 & 320 & 320 \\
\hline \hline \multicolumn{2}{l}{ Robust standard errors in parentheses. ${ }^{*} p<0.10,{ }^{* *} p<0.05,{ }^{* * *} p<0.01$}
\end{tabular}

Table 3 splits the dataset between the first and last 10 rounds. Here we find that, in the first 10 rounds, both players 2 and 3 benefit from player 1's deviation. However, in the second half of the experiment only player 2 gains, and twice as much with respect to the first half. These results suggest that the learning effects enhance player 2's positional advantage over player 3 in the continuation of the game.

Table 3: Regression of winning probability on specific strategy in PS

\begin{tabular}{lcccccc}
\hline \hline & \multicolumn{3}{c}{ First 10 rounds } & \multicolumn{3}{c}{ Last 10 rounds } \\
\hline & Player1 & Player2 & Player3 & Player1 & Player2 & Player3 \\
\hline Player 1 deviates & $-0.324^{* * *}$ & $0.199^{* *}$ & $0.134^{* *}$ & $-0.337^{* * *}$ & $0.387^{* * *}$ & -0.049 \\
& $(0.077)$ & $(0.091)$ & $(0.068)$ & $(0.047)$ & $(0.059)$ & $(0.071)$ \\
Constant & & & & & & \\
& $0.597^{* * *}$ & $0.290^{* * *}$ & $0.108^{* * *}$ & $0.570^{* * *}$ & $0.230^{* * *}$ & $0.200^{* * *}$ \\
& $(0.062)$ & $(0.051)$ & $(0.032)$ & $(0.022)$ & $(0.037)$ & $(0.039)$ \\
\hline Obs. & 160 & 160 & 160 & 160 & 160 & 160 \\
\hline \hline
\end{tabular}

Robust standard errors in parentheses. ${ }^{*} p<0.10,{ }^{* *} p<0.05,{ }^{* * *} p<0.01$ 


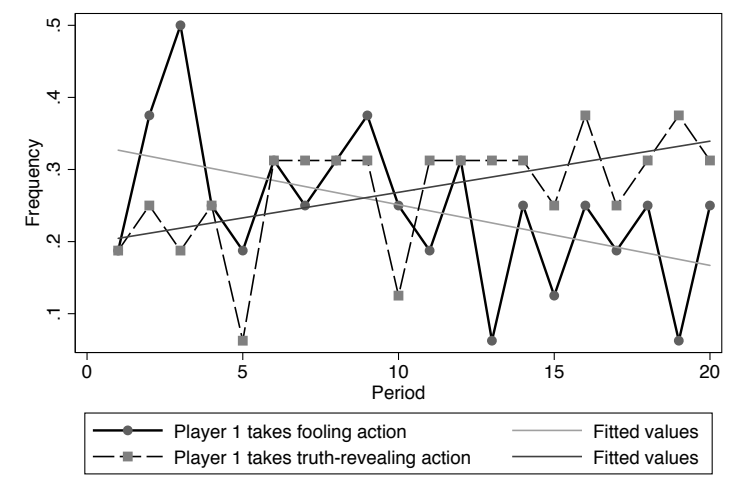

Figure 3: Player 1's off-equilibrium behavior in CS

\section{- CS: Does Fooling Work?}

In CS we focus on the effects of strategic manipulation. In this context, "fooling" on behalf of player 1 is defined as a guessing strategy which is incompatible with the realized signal, namely,

$$
g_{1}=\left\{\begin{array}{l}
3, \text { if } s_{1}=0 \\
0, \text { if } s_{1}=1
\end{array}\right.
$$

We find that $24.7 \%(79 / 320)$ of choices of player 1 fits this definition. At the individual level, the frequencies of fooling for the 16 subjects acting as player 1 in CS ranges from $0 \%$ to $50 \%$, with a median of $30 \%$.

By the same token, "revealing" on behalf of player 1 is defined as the equilibrium guessing strategy in PS, namely, if $g_{1}=s_{1}+2$. We find that $22 \%(72 / 320)$ of choices of player 1 fits into this category. The individual frequencies of revealing for the 16 subjects acting as player 1 range from $5 \%$ to $45 \%$, with a median of $22 \%$. Figure 3 tracks the relative frequencies of use of either strategy across the 20 rounds. As Figure 3 shows, there is an increasing (decreasing) trend in the frequencies of use of the revealing (fooling) strategies, respectively.

To analyze the effects of player 1's signaling on winning probabilities, we estimate the following random-effect linear probability model:

$$
P\left(y_{i t}=1\right)=\alpha+\beta_{1} x_{1 t}+\beta_{2} z_{1 t}+u_{i t},
$$

where $y_{i t}$ is a binary index which is positive if player $i$ wins the prize at round $t$ and $x_{1 t}\left(z_{1 t}\right)$ is positive if player 1 uses the fooling (revealing) strategy, respectively. Table 4 reports the estimation results. As Table 4 shows, if player 1 uses fooling strategy, her own winning probability increases by $15 \%$, mostly at the expense of player 3 . As for player 2, she seems to benefit from player 1's fooling. This suggests that player 2 decodes player 1's signals better than player 3. By contrast the adoption on behalf of player 1 of a revealing strategy has no significant effect on any player's winning probability. 
Table 4: Decoding and learning dynamics in CS (I)

\begin{tabular}{lccc}
\hline \hline & Player1 & Player2 & Player3 \\
\hline Player 1 fools & $0.152^{* *}$ & $0.088^{*}$ & $-0.219^{* *}$ \\
& $(0.068)$ & $(0.052)$ & $(0.094)$ \\
Player 1 reveals & -0.000 & 0.031 & -0.025 \\
& $(0.059)$ & $(0.056)$ & $(0.087)$ \\
Constant & $0.253^{* * *}$ & $0.129^{* * *}$ & $0.611^{* * *}$ \\
& $(0.032)$ & $(0.031)$ & $(0.053)$ \\
\hline Obs. & 320 & 320 & 320 \\
\hline \hline Robust standard errors in parentheses. $* p<0.10^{* *} p<0.05, * * *<0.01$
\end{tabular}

Once again, Table 5 splits the dataset into the first (last) 10 rounds, respectively, to look for learning effects. As Table 5 shows, benefits from fooling for player 1 are limited to the first periods only, and disappear as the experiment proceeds. In the meantime, player 2 (much more than player 3 ) seems to learn on how to decode player 1's fooling, while player 3's performance does not improve. If player 1 reveals, this has no significant effects on anyone's winning expectations.

To summarize: the results for the full sample seem mostly driven by what happens in the first 10 rounds. In the second half the experiment, the pattern changes dramatically, in that fooling does not seem sustainable in the long-run.

Table 5: Decoding and learning dynamics in CS (II)

\begin{tabular}{lcccccc}
\hline \hline & \multicolumn{3}{c}{ First 10 rounds } & \multicolumn{4}{c}{ Last 10 rounds } \\
& Player1 & Player2 & Player3 & Player1 & Player2 & Player3 \\
\hline Player 1 fools & $0.230^{* * *}$ & -0.009 & $-0.216^{* *}$ & 0.071 & $0.215^{* * *}$ & $-0.222^{*}$ \\
& $(0.087)$ & $(0.059)$ & $(0.110)$ & $(0.105)$ & $(0.077)$ & $(0.123)$ \\
Player 1 reveals & 0.083 & -0.079 & 0.001 & -0.076 & $0.118^{* *}$ & -0.027 \\
& $(0.103)$ & $(0.082)$ & $(0.140)$ & $(0.061)$ & $(0.059)$ & $(0.083)$ \\
Constant & & & & & & \\
& $0.187^{* * *}$ & $0.165^{* * *}$ & $0.646^{* * *}$ & $0.316^{* * *}$ & $0.097^{* *}$ & $0.570^{* * *}$ \\
\hline Obs & $(0.044)$ & $(0.053)$ & $(0.080)$ & $(0.051)$ & $(0.041)$ & $(0.072)$ \\
\hline \hline Robust standard errors in parentheses. ${ }^{*} p<0.10,{ }^{* *} p<0.05,{ }^{* * *} p<0.01$ & 160 & 160 \\
\hline
\end{tabular}




\begin{tabular}{|c|c|c|}
\hline Variables & Player 1 & Player 3 \\
\hline \multirow{2}{*}{$\overline{c\left(g_{1}, s_{1}\right)^{+}}$} & 0.189 & -0.0509 \\
\hline & $(0.221)$ & $(0.323)$ \\
\hline \multirow[t]{2}{*}{$c\left(g_{1}, s_{1}\right)^{-}$} & $-0.552 * *$ & $0.662 *$ \\
\hline & $(0.207)$ & $(0.302)$ \\
\hline \multirow{2}{*}{$c\left(g_{2}, s_{2}\right)$} & $-0.217^{*}$ & 0.142 \\
\hline & $(0.0983)$ & $(0.144)$ \\
\hline \multirow[t]{2}{*}{$c\left(g_{3}, s_{3}\right)$} & $-0.403 * *$ & $0.568^{* *}$ \\
\hline & $(0.122)$ & $(0.178)$ \\
\hline \multirow[t]{2}{*}{$c\left(g_{2}, g_{1}\right)$} & 0.107 & -0.0713 \\
\hline & $(0.0763)$ & $(0.112)$ \\
\hline \multirow{2}{*}{$c\left(g_{3}, g_{1}\right)$} & -0.0404 & -0.00722 \\
\hline & $(0.0822)$ & $(0.120)$ \\
\hline \multirow[t]{2}{*}{$c\left(g_{3}, g_{2}\right)$} & 0.185 & 0.223 \\
\hline & $(0.142)$ & $(0.207)$ \\
\hline \multirow[t]{2}{*}{ Constant } & $0.345^{* * *}$ & $0.284^{*}$ \\
\hline & $(0.0896)$ & $(0.131)$ \\
\hline Obs. & 16 & 16 \\
\hline
\end{tabular}

Table 6: Identifying the effect of fooling from revealing in CS 